\title{
HYDRODYNAMIC COEFFICIENTS FOR SLENDER CIRCULAR COLUMNS OSCILLATING UNDER SINUSOIDAL WAVE ACTION
}

\author{
By K. VENKATARAMANA*, Susumu YOSHIHARA** and Yoshikazu YAMADA***
}

\begin{abstract}
This paper discusses the experimental determination of hydrodynamic coefficients of Morison equation for slender circular columns oscillating under sinusoidal wave action. Two types of columns are considered for study. Column-1 is a rigid circular column, hinged at the sea bed and the response oscillation is in the form of angular displacement. Column- 2 is a flexible circular column, fixed at sea bed and the response oscillation is in the form of structural deflection. The columns are oscillated by sinusoidal waves in a two-dimensional wave tank and the responses are recorded. The hydrodynamic coefficients associated with the wave force equation are determined and are plotted against Keulegan-Carpenter number. The results are compared with the values given by other researchers and show a fairly good agreement.

Keywords: Morison equation, hydrodynamic coefficients, response oscillation
\end{abstract}

\section{INTRODUCTION}

The empirical force model known as the Morison equation ${ }^{1)}$ is widely used for estimating the wave forces on slender offshore columns. This equation assumes that the columns are stationary and the incident wave field is not disturbed by the presence of columns. However, in real situations, slender columns are likely to oscillate due to incident waves and create disturbance in the surrounding wave field. In particular, the amplitude of oscillation is large for structures such as guyed towers which have relatively large natural periods. The Morison equation is generalized for non-stationary columns by considering the relative motion between the water particles and the columns and is given as ${ }^{2)}$

$$
F(z, t)=C_{M} \rho \pi r_{0}^{2} \frac{\partial u}{\partial t}-C_{m} \rho \pi r_{0}^{2} \frac{\partial^{2} x}{\partial t^{2}}+C_{D} \rho r_{0}\left(u-\frac{\partial x}{\partial t}\right)\left|\left(u-\frac{\partial x}{\partial t}\right)\right|
$$

in which $F(z, t)$ is the horizontal wave force per unit length of the column at depth $z$ and time $t, C_{M}$ is the inertia coefficient, $C_{m}$ is the added mass coefficient, $C_{D}$ is the drag coefficient, $\rho$ is the mass density of water, $r_{0}$ is the radius of the column, $u$ and $\partial u / \partial t$ are the horizontal water particle velocity and acceleration respectively at the center position if the column were not present, $x$ is the response displacement of the column, $\partial x / \partial t$ and $\partial^{2} x / \partial t^{2}$ are the corresponding velocity and acceleration respectively of the column. The hydrodynamic coefficients associated with the above wave force equation are determined experimentally either by oscillating the structure in a still fluid ${ }^{3) .4}$ or by oscillating the fluid past a stationary structure ${ }^{5)(6)}$.

The objective of this paper is to investigate the values of the hydrodynamic coefficients when both the

* Member of JSCE, M. Eng., Graduate Student, Dept. of Civil Engineering, Kyoto University, Kyoto City 606, Japan.

** Member of JSCE, Dr. Eng., Professor, Dept. of Ocean Civil Engineering, Kagoshima University.

*** Member of JSCE, Dr. Eng., Professor, Dept. of Civil Engineering, Kyoto University. 
fluid and the structure are oscillating. Emphasis is placed on the evaluation of hydrodynamic coefficients near the resonance point. Small amplitude wave theory is used to describe the wave kinematics. Two types of columns are considered for study. Column-1 is a rigid circular column, hinged at sea bed and the response oscillation is in the form of angular displacement. Column-2 is a flexible circular column, fixed at sea bed and the response oscillation is in the form of structural deflection. Both the columns are treated as single-degree-of-freedom systems. The columns are oscillated under sinusoidal waves in a two-dimensional wave tank. The measured wave elevation and in-line responses of the columns are used to obtain the inertia, added mass and drag coefficients. These hydrodynamic coefficients are plotted as functions of the corresponding Keulegan-Carpenter (K-C) parameter.

\section{EXPRESSIONS FOR HYDRODYNAMIC COEFFICIENTS}

\section{(1) Column-1}

Fig. 1 is the schematic diagram of Column-1 in equilibrium under wave action. The rotational displacement of the column $\beta(t)$ at any time $t$ is assumed to be small so that $\tan (\beta)=\beta$. Applying the principles of dynamic equilibrium, the equation of motion for the column is obtained as

$$
\frac{m l^{2}}{3} \frac{d^{2} \beta}{d t^{2}}+C_{0} \frac{d \beta}{d t}+\left(\frac{B h}{2}-\frac{m g l}{2}\right) \beta=\int_{-h}^{0} F(h+z) d z
$$

where $m$ is the mass of the column, $l$ is the column length, $B$ is the buoyancy force on the column, $h$ is the depth of water below still water level, $g$ is the acceleration due to gravity, $d \beta / d t$ and $d^{2} \beta / d t^{2}$ are respectively the angular velocity and the angular acceleration of the column at any time $t$ and $C_{0}$ is the structural damping coefficient in

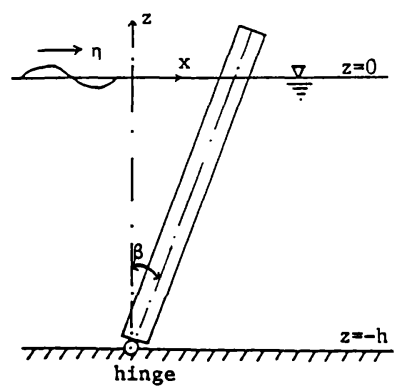

Fig. 1 Schematic diagram of Column-1. water. By substituting Eq. (1) in the right hand side of Eq. (2) and integrating, moment of wave force for progressive sinusoidal wave action is obtained. The nonlinear drag term in Eq. (1) is linearized in the classical manner ${ }^{7)}$ by equalizing the work done in a wave period by the nonlinear drag force with that by the equivalent linearized drag force, i. e.,

$$
\begin{aligned}
& \left(u-\frac{\partial x}{\partial t}\right)\left|\left(u-\frac{\partial x}{\partial t}\right)\right|=\left[H^{\prime} \exp (i \omega t)-(h+z) \frac{d \beta}{d t}\right]\left|H^{\prime} \exp (i \omega t)-(h+z) \frac{d \beta}{d t}\right| \\
& =C_{e q}\left[H^{\prime} \exp (i \omega t)-(h+z) \frac{d \beta}{d t}\right]
\end{aligned}
$$

where $\quad C_{e q}=\frac{8}{3 \pi}\left|H^{\prime}+i \omega(h+z) \beta_{0} \exp (-i \psi)\right|, H^{\prime}=\frac{g k a}{\omega} \frac{\cosh [k(h+z)]}{\cosh (k h)}$, $\beta=\beta_{0} \exp [i(\omega t-\psi)]$

where $a$ is the wave amplitude, $k$ is the wave number, $\omega$ is the circular wave frequency, $\beta_{0}$ is the amplitude of angular response displacement and $\psi$ is the phase difference between the water level elevation and the angular displacement. Finally, the equation of motion becomes

$$
\frac{d^{2} \beta}{d t^{2}}+2 h_{0} \omega_{0} \frac{d \beta}{d t}+\frac{C_{D} C_{1}}{M} \frac{d \beta}{d t}\left|\frac{d \beta}{d t}\right|+\omega_{0}^{2} \beta=\left[\frac{i C_{M} F_{M}+C_{D} F_{D}}{M}\right] \exp (i \omega t)
$$

where $\quad M=M_{0}+C_{m} M_{A}, \quad M_{0}=\frac{m l^{2}}{3}, M_{A}=\rho \pi r_{0}^{2} \frac{h^{3}}{3}, C_{1}=\frac{2 \rho r_{0} h^{4}}{3 \pi}, \quad \omega_{0}=\sqrt{\frac{B h-m g l}{2\left(M_{0}+C_{m} M_{A}\right)}}$

$$
\begin{aligned}
& F_{M}=w \pi r_{0}^{2} h a\left[\frac{k h \sinh (k h)-\cosh (k h)+1}{k h \cosh (k h)}\right], \\
& F_{D}=w r_{0} h a^{2}\left[\frac{2\left\{2 k^{2} h^{2}+1+2 k h \sinh (2 k h)-\cosh (2 k h)\right\}}{3 \pi k h \sinh (2 k h)}\right]
\end{aligned}
$$

in which $h_{0}$ is the equivalent viscous damping ratio, $\omega_{0}$ is the natural frequency of vibration in water and $w$ 
is the unit weight of water. The expression for the added mass coefficient $C_{m}$ is

$$
C_{m}=\frac{1}{M_{A}}\left[\frac{B h-m g l}{2 \omega_{0}^{2}}-M_{0}\right]
$$

Now using $\beta=\beta_{0}[\cos (\psi)-i \sin (\psi)] \exp (i \omega t)$ and equating the real and the imaginary parts, expressions for the drag and the inertia coefficients are

$$
\begin{aligned}
& C_{D}=\frac{M\left[\left(\omega_{0}^{2}-\omega^{2}\right) \beta_{0} \cos (\psi)+2 h_{0} \omega_{0} \omega \beta_{0} \sin (\psi)\right]}{F_{D}+C_{1} \omega^{2} \beta_{0}^{2} \cos (2 \psi)} \\
& C_{M}=\frac{M\left[\left(\omega^{2}-\omega_{0}^{2}\right) \beta_{0} \sin (\psi)+2 h_{0} \omega_{0} \omega \beta_{0} \cos (\psi)\right]+C_{D} C_{1} \omega^{2} \beta_{0}^{2} \sin (2 \psi)}{F_{M}}
\end{aligned}
$$

\section{(2) Column-2}

Fig. 2 shows the column considered for study. The response displacement $x(z, t)$ of the column at any depth $z$ and time $t$, for steady vibration under sinusoidal waves, may be expressed as

$$
x(z, t)=\phi(z) x_{t}(t)=\phi(z) X_{t} \exp [i(\omega t-\psi)]
$$

where $\phi(z)=\frac{3(h+z)^{2}}{2 l^{2}}-\frac{(h+z)^{3}}{2 l^{3}}$

where $\phi$ is the shape function for the column, $x_{t}(t)$ is the response displacement of the column top at time $t, X_{t}$ is the amplitude of the response displacement and $\psi$ is the phase difference between the water elevation and the steady displacement of the column top.

The equation of motion is formulated in terms of generalized co-ordinates using the Hamilton's energy principle ${ }^{8)}$ and is obtained as

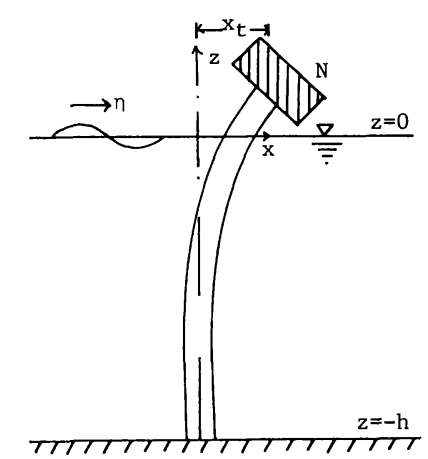

Fig. 2 Schematic diagram of Column-2.

$$
\left[\frac{33}{140} m+\frac{N}{g}\right] \frac{d^{2} x_{t}}{d t^{2}}+C_{0} \frac{d x_{t}}{d t}+\left[\frac{3 E I}{l^{3}}-\frac{6 N}{5 l}\right] x_{t}=\int_{-h}^{0} F \phi d z
$$

where $N$ is the mass of the super-imposed load (platform mass), $E$ is the Young's modulus of elasticity of the column material and $I$ is the moment of inertia of the column cross section.

By substituting Eq. (1) in the right hand side of Eq. (9) and integrating, the expression for the generalized wave force is obtained. The nonlinear drag term is linearized in the same manner as stated previously. Finally, the equation of motion becomes

$$
\frac{d^{2} x_{t}}{d t^{2}}+2 h_{0} \omega_{0} \frac{d x_{t}}{d t}+\frac{C_{D} C_{1}}{M} \frac{d x_{t}}{d t}\left|\frac{d x_{t}}{d t}\right|+\omega_{0}^{2} x_{t}=\left[\frac{i C_{M} F_{M}+C_{D} F_{D}}{M}\right] \exp (i \omega t)
$$

where $M=M_{0}+C_{M} M_{A}, \quad M_{0}=\frac{33}{140} m+\frac{N}{g}, M_{A}=\rho \pi r_{0}^{2} h \frac{\alpha^{4}\left(5 \alpha^{2}-35 \alpha+63\right)}{140}, \alpha=\frac{h}{l}$

$$
\begin{aligned}
& C_{1}=\rho r_{0} h \frac{\alpha^{6}\left(-28 \alpha^{3}+280 \alpha^{2}-945 \alpha+1080\right)}{840 \pi}, \omega_{0}=\sqrt{\frac{\frac{3 E I}{l^{3}-\frac{6 N}{5}}}{M_{0}+C_{m} M_{A}}} \\
& F_{M}=w \pi r_{0}^{2} a \frac{\left.\alpha^{2}\left\{\left[k^{3} h^{3}(3-\alpha)+6 k h(1-\alpha)\right] \sinh (k h)+\left[6 \alpha-3 k^{2} h^{2}(2-\alpha)\right] \cosh (k h)-6 \alpha\right]\right\}}{2 k^{3} h^{3} \cosh (k h)}
\end{aligned}
$$$$
F_{D}=w r_{0} a^{2} \frac{\left[4 k^{3} h^{3}(3-\alpha)+6 k h(1-\alpha)\right] \sinh (2 k h)}{6 \pi \alpha k^{3} l^{3}} *
$$

$$
* \frac{+\left[3 \alpha-6 k^{2} h^{2}(2-\alpha)\right] \cosh (2 k h)-3 \alpha+2 \alpha(4-\alpha) k^{3} h^{3}}{\sinh (2 k h)}
$$

The expressions for the hydrodynamic coefficients are obtained as 


$$
\begin{aligned}
& C_{m}=\frac{1}{M_{A}}\left[\frac{\left\{\frac{3 E I}{l^{3}}-\frac{6 N}{5 l}\right\}}{\omega_{0}^{2}}-M_{0}\right] \\
& C_{D}=\frac{M\left[\left(\omega_{0}^{2}-\omega^{2}\right) X_{t} \cos (\psi)+2 h_{0} \omega_{0} \omega X_{t} \sin (\psi)\right]}{F_{D}+C_{1} \omega^{2} X_{t}^{2} \cos (2 \psi)} \\
& C_{M}=\frac{M\left[\left(\omega^{2}-\omega_{0}^{2}\right) X_{t} \sin (\psi)+2 h_{0} \omega_{0} \omega X_{t} \cos (\psi)\right]+C_{D} C_{1} \omega^{2} X_{t}^{2} \sin (2 \psi)}{F_{M}}
\end{aligned}
$$

The columns are oscillated sinusoidally by applying periodic waves of amplitude $a$. The amplitude of the displacement (amplitude of angular displacement for Column-1 and amplitude of horizontal displacement of column top for Column-2) and its phase difference with the wave elevation are measured. By substituting those values in the appropriate equations, the hydrodynamic coefficients are determined.

\section{DETAILS OF THE EXPERIMENTS}

Experiments were carried out in a twodimensional wave tank of $30 \mathrm{~m}$ long, $1 \mathrm{~m}$ wide and $1.2 \mathrm{~m}$ deep. Depth of water in the tank during the experiments was $0.75 \mathrm{~m}$. The test models were hollow cylindrical columns made of poly-vinyl chloride plastic. The dimensions of the columns are given in Table 1. Regular waves were generated by a flap type wave generator and the energy of the waves was dissipated at the other end of the tank using an armour block-mesh beach, inclined to the water surface.

Table 1 Details of the column models.

\begin{tabular}{|l|c|c|c|}
\hline \multirow{2}{*}{} & \multirow{2}{*}{ Column-1 } & \multicolumn{2}{|c|}{ Column-2 } \\
\cline { 3 - 4 } & & Model-1 & Model-2 \\
\hline Outer diameter (mm) & 60.0 & 38.2 & 60.2 \\
Inner diameter (mm) & 56.6 & 30.6 & 50.8 \\
Length (m) & 0.9 & 0.928 & 0.928 \\
Self weight (N/m) & 5.24 & 5.34 & 11.0 \\
Super-imposed load(kN) & -- & 0.129 & 0.561 \\
Flexural rigidity (kN.m) & 32.92 & 15.29 & 79.09 \\
Natural period of vibration & 1.88 & 1.14 & 1.009 \\
in water (sec) & & $2 \%$ & $2 \%$ \\
Damping ratio in water & $5 \%$ & $2 \%$ & 1.06 \\
Added mass coefficient $\mathrm{C}_{\mathrm{m}}$ & 0.84 & 0.97 & \\
\hline
\end{tabular}

\section{(1) Column-1}

The base of the column was hinged to the floor near the middle of the wave tank. The hinge was so designed that it allowed the rotation of the column only in the wave propagation direction. The steady dynamic response displacement of the column under sinusoidal wave action was measured using a displacement measuring device called 'Non-contact Electro-Optical Displacement Follower'. (This instrument consists of an optical camera and a control unit. The optical camera catches the movements of the model. The light passing through the aperture depends on the position of the image. The smaller the displacement of the model, the lower is the amount of light passing through the aperture. This light is converted into electrons by means of the aperture electrodes and the displacement is measured in the control unit in volts. The special features of this instrument are : (1) all movements of the model are analyzed without contacting the model thereby not loading or changing the dynamics of the model, (2) The dynamic displacement of the model can be recorded in the time scale for single axis oscillations or biaxial oscillations.) The movement of the water surface was measured by a capacitance type wave sensor positioned at the neutral position of the column and its electrical output was recorded in the time scale in syncronization with the output of the control unit of the displacement measuring instrument. All the data was initially recorded in a data recorder. Subsequently, the discrete data was obtained by the analog to digital conversion of the recored data using an A-D converter system. Experiments were conducted for periodic waves of $30 \mathrm{~mm}$ and $60 \mathrm{~mm}$ wave heights and wave frequencies ranging from 0.2 to $1.2 \mathrm{cycles} / \mathrm{sec}$.

( 2 ) Column-2

The base of the column was fixed to the floor near the middle of the tank. The response of the column to regular waves was measured using a servo-type accelerometer placed on the column top in-line with the 
wave propagation direction. The movement of the water surface was measured by a capacitance type wave sensor positioned at the neutral position of the column and its electrical output was recorded by a pen oscilloscope in the time scale in syncronization with the output of the accelerometer. Experiments were carried out for periodic waves of $30 \mathrm{~mm}$ and $60 \mathrm{~mm}$ wave heights and wave frequencies ranging from 0.7 to $1.3 \mathrm{cycles} / \mathrm{sec}$.

\section{DATA ANALYSIS AND DISCUSSIONS}

During free vibration experiments in still water, the columns oscillated about their neutral position with constant circular frequencies. These frequencies are nearly equal to their natural frequencies. The magnitude of the response seemed to diminish exponentially. Damping ratios were calculated from the response peaks of the free vibration curves. The results are tabulated in Table 1 together with the values of the added mass coefficient obtained from Eqs. (5) and (11).

Wave amplitude, wave frequency, amplitude of response displacement (Column-1) or amplitude of response acceleration (Column-2), and phase difference between the wave elevation and the response were read from the recorded data of the forced vibration experiments. Figs. 3 and 5 show the resonance curves and the phase difference curves. The values of hydrodynamic coefficients $C_{M}$ and $C_{D}$ are plotted against $\mathrm{K}-\mathrm{C}$ number in Figs. 4 and 6 . (The range of $\mathrm{K}-\mathrm{C}$ number covered in the present experiment is relatively small due to practical limitations. ) It is seen that the values scatter around 2.0 for $C_{M}$ and 1.0 for $C_{D}$ which are the usually adopted values. As a matter of comparison, Figs. 7 and 8 show the values of these coefficients for a stationary structure in an oscillating fluid ${ }^{5}$ (mean polinominal curves are fitted through experimental data) and for an oscillating structure in a still fluid ${ }^{3)}$ respectively.

\section{CONCLUSIONS}

Morison coefficients have been determined experimentally for slender circular columns oscillating under sinusoidal wave action. The values scatter around $C_{M}=2.0$ and $C_{D}=1.0$ which are the usually adopted values in the engineering practice. The results are compared with the corresponding values for a stationary
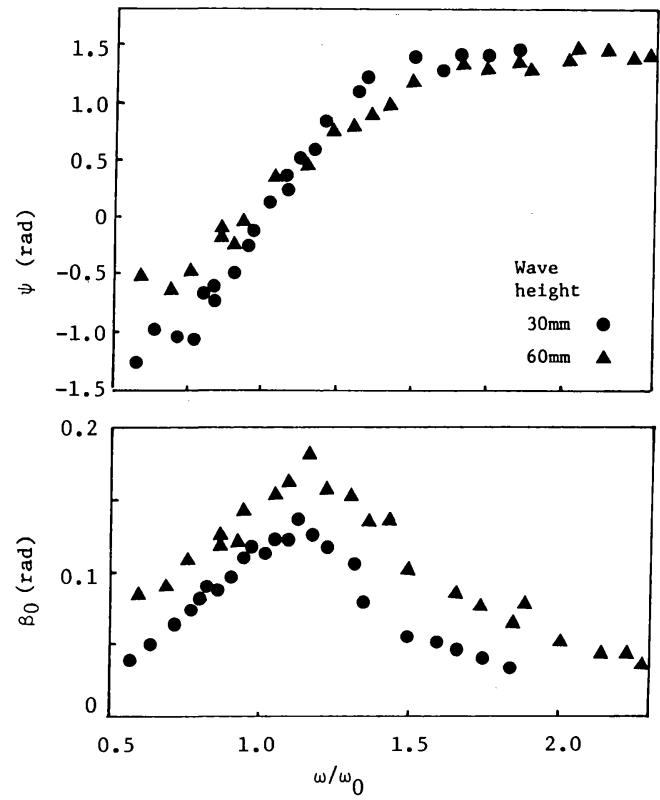

Fig. 3 Amplitude of response displacement $\beta_{0}$ and phase difference $\psi$ between wave elevation and response displacement (Column-1).
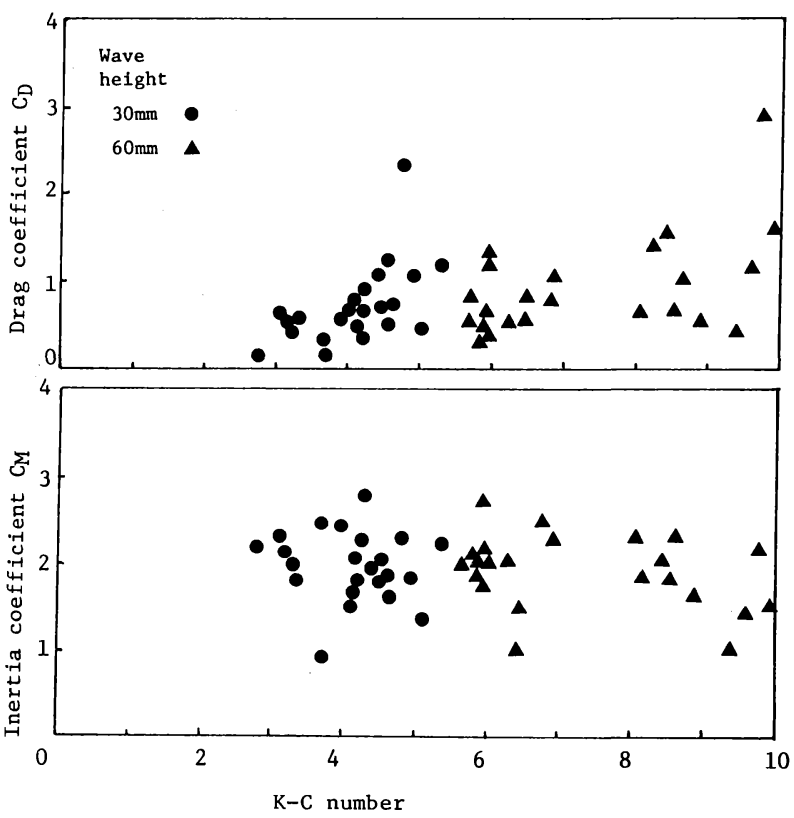

Fig. 4 Hydrodynamic coefficients $C_{M}$ and $C_{D}$ (Column-1). 

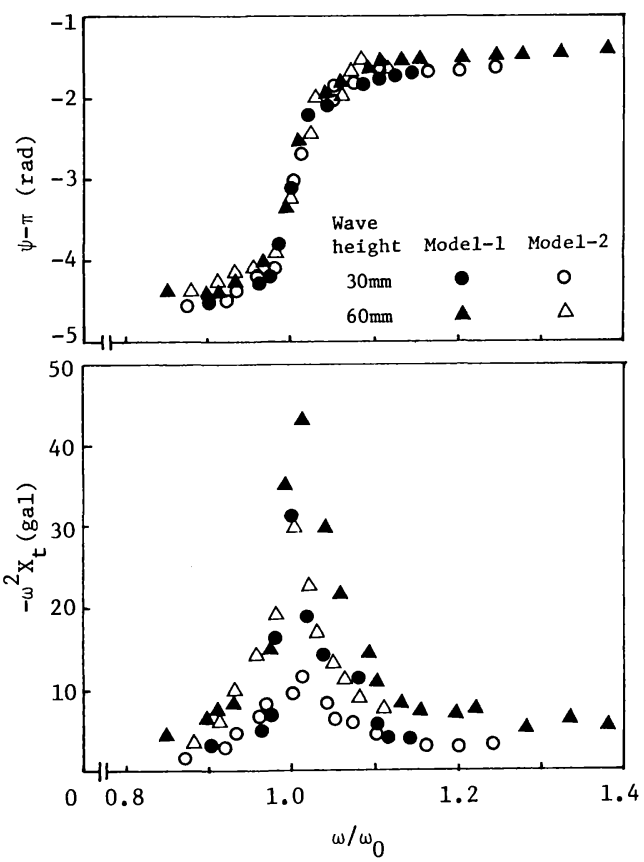

Fig. 5 Amplitude of response acceleration $-\omega^{2} X_{t}$ and phase difference $\psi-\pi$ between wave elevation and response acceleration (Column-2).

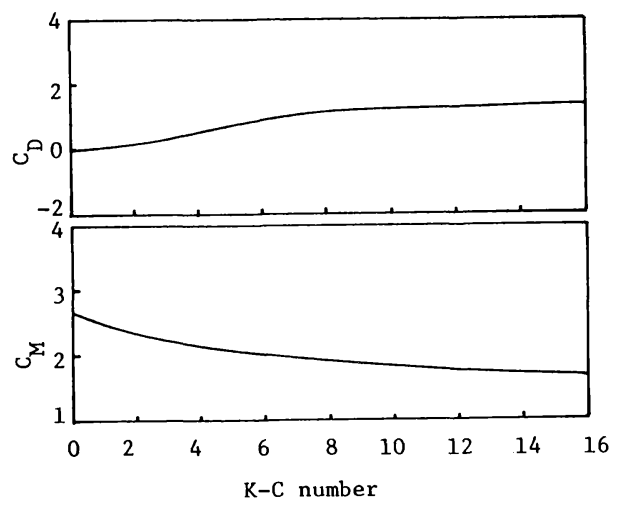

Fig. 7 Hydrodynamic coefficients $C_{M}$ and $C_{D}$ for a stationary structure in oscillating fluid (Chakrabarti et al. ${ }^{5)}$ ).

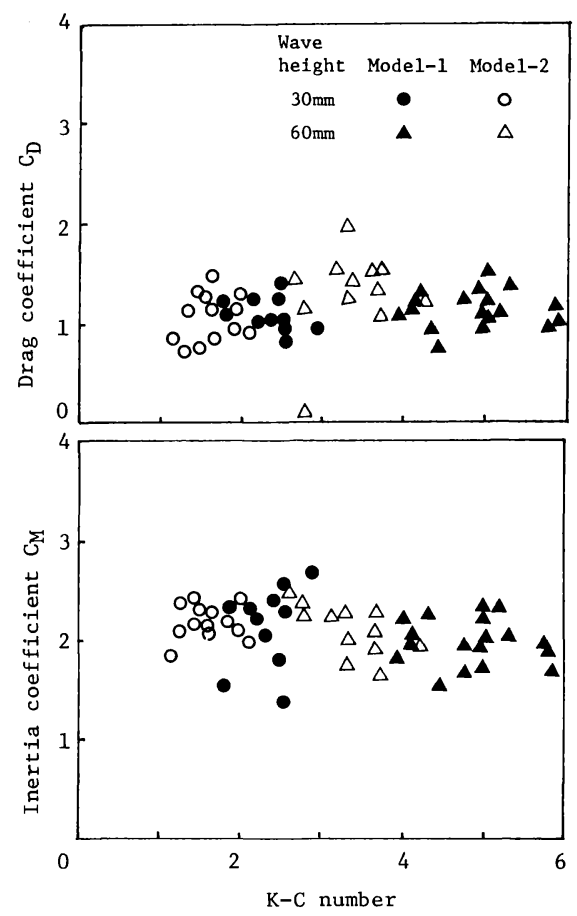

Fig. 6 Hydrodynamic coefficients $C_{M}$ and $C_{D}$ (Column-2).

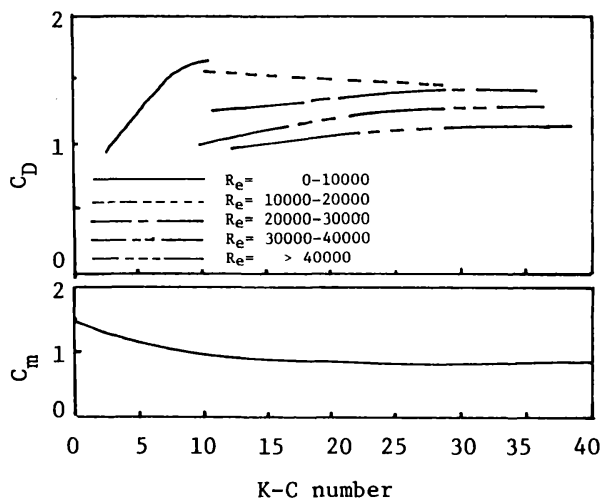

Fig. 8 Hydrodynamic coefficients $C_{m}\left(C_{M}=1+C_{m}\right)$ and $C_{D}$ for an oscillating structure in a still fluid (Chakrabarti et al. ${ }^{3)}$ ).

structure in an oscillating fluid, and for an oscillating structure in a still fluid and show a fairly good agreement.

\section{REFERENCES}

1) Morison, J. R., et al. : The Force Exerted by Surface Waves on Piles, Petroleum Trans., AIME, Vol. 189, pp. 149-154, 1950.

2) Brebbia, C. A. : Fluid Structure Interaction Problems, Vibrations of Engineering Structures, C. A. Brebbia, et al., ed., Springer-Verlag, Berlin, 1985.

3) Chakrabarti, S. K., et al. : Hydrodynamic coefficients of a harmonically oscillated tower, Applied Ocean Research, Vol.5, No. 4, pp. 226-233, 1983.

4) Garrison, C. J., et al. : Drag and Inertia forces on a cylinder in a periodic flow, Journal of the Waterway, Port, Coastal and 
Ocean Engineering Division, ASCE, Vol. 103, No. WW 2, pp. 193-204, 1977.

5) Chakrabarti, S. K., et al. : Wave Forces on Vertical Circular Cylinder, Journal of the Waterways, Harbors, and Coastal Engineering Division, ASCE, Vol. 102, No. WW 2, pp. 203-221, 1976.

6) Sarpkaya, T. : Force on a circular cylinder in viscous oscillatory flow at low Keulegan-Carpenter numbers, Journal of Fluid Mechanics, Vol. 165, pp. 61-71, 1986.

7) Dahong, Q. and Qihua, Z. : The dynamic response of an underwater column hinged at the sea bottom under the wave action, Offshore Technology Conference, pp. 523-531, 1982.

8) Clough, R. W. and Penzien, J. : Dynamics of Structures, McGraw-Hill Kogakusha Ltd., Tokyo, pp. 17-40, 1975. 